\title{
Progress in differential deposition for improving the figures of full-shell astronomical grazing incidence $X$-ray optics
}

\footnotetext{
* Kiranmayee Kilaru, USRA/NASA MSFC

* Carolyn Atkins, University of Alabama in Huntsville/NASA MSFC

* Brian D. Ramsey, NASA MSFC

* Jeffery Kolodziejczak, NASA MSFC

* Mikhail V. Gubarev, NASA MSFC

* Stephen L. O’Dell, NASA MSFC

* David M. Broadway, NASA MSFC
} 


\section{Why differential deposition?}

Typical mid-spatial

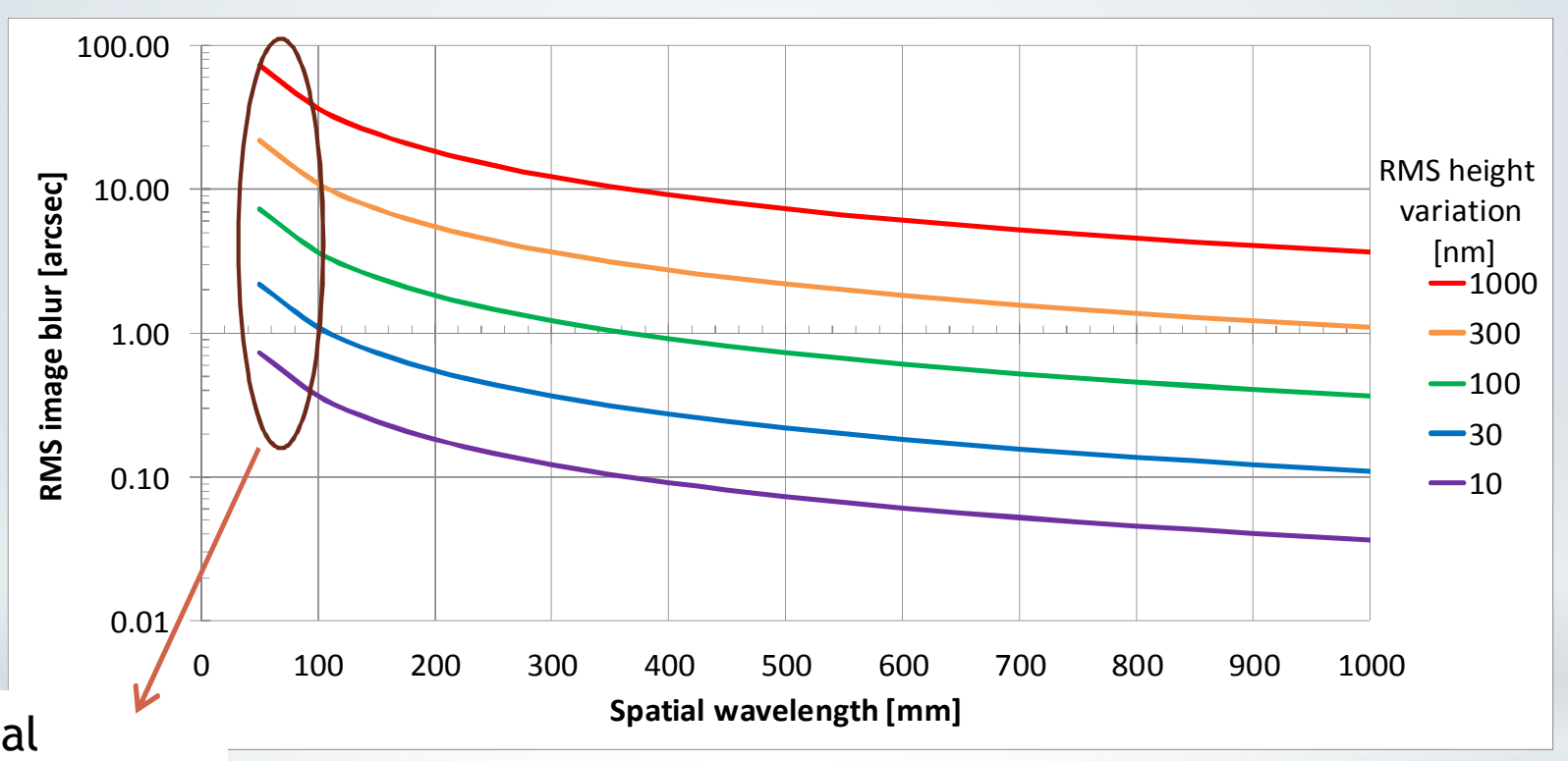

frequency range in X-ray

mirrors

* Imaging quality of X-ray optics can be significantly improved if the RMS height variations can be reduced 


\section{Concept of differential deposition}

* Use of physical vapor deposition to selectively deposit material on the mirror surface to smooth out figure imperfections

* Various approaches -

* A) Constant mirror velocity- varying slit width

* B) Varying mirror translation velocity

* C) Varying power on target material

* D) Combination of above all SPIE SanDiego 2015

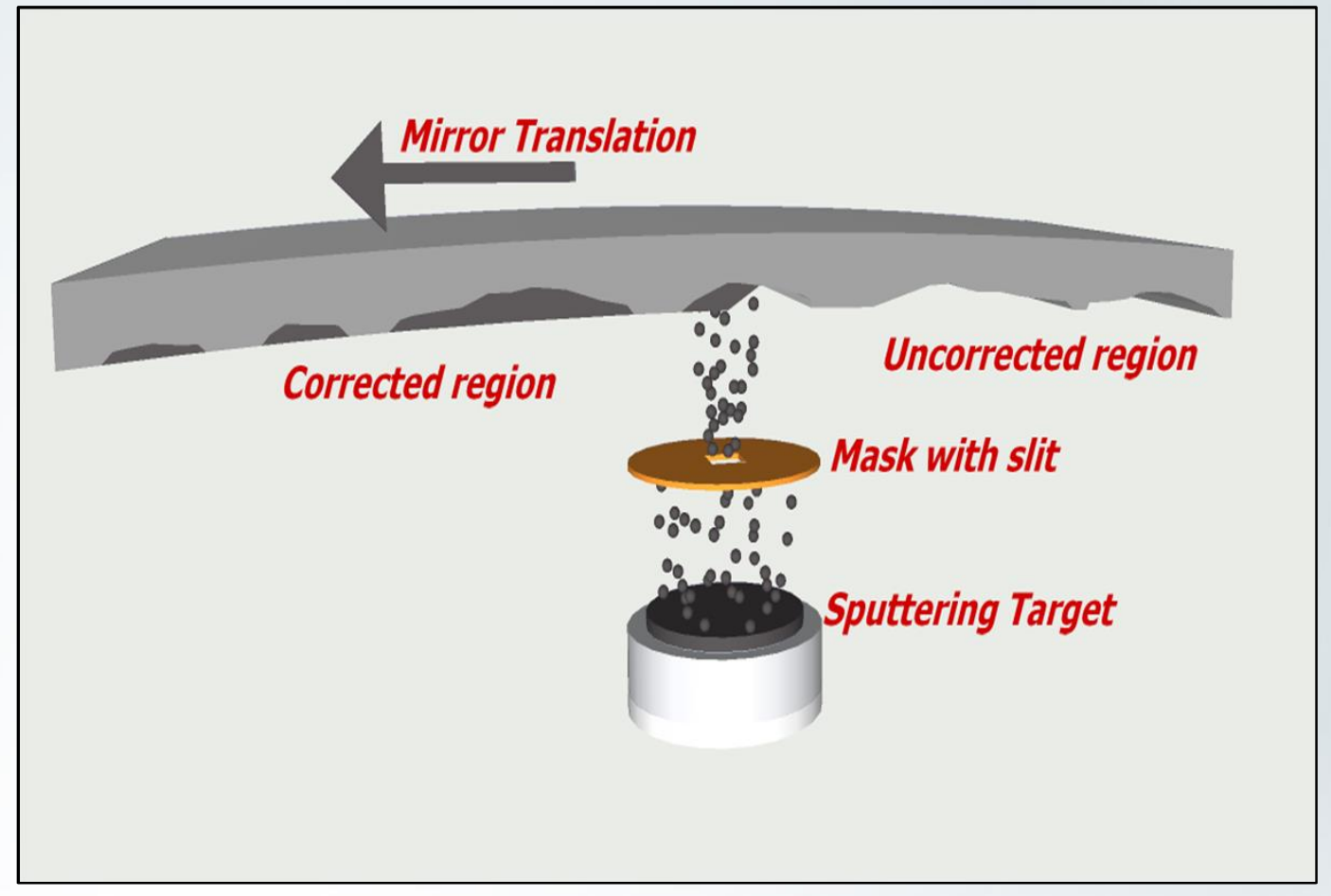

Ice, G. E., Chung, J. S., Tischler, J. Z., Lunt, A., and Assoufid, L., "Elliptical x-ray microprobe mirrors by differential deposition”, Rev. Sci. Instr., 71(7), 2635-2639 (2000).

* Handa Soichiro, Hidekazu Mimura, Hirokatsu Yumoto, Takashi Kimura, Satoshi Matsuyama, Yasuhisa Sano, Kazuto Yamauchi, "Highly accurate differential deposition for X-ray reflective optics", Surface and Interface Analysis, 40, 1019-1022 (2008).

* Alcock, S. G., and S. Cockerton. "A preferential coating technique for fabricating large, high quality optics." Nuclear Instruments and Methods in Physics Research Section A: Accelerators, Spectrometers, Detectors and Associated Equipment 616, no. 2 (2010): 110-114.

* Two-dimensional differential deposition for figure correction of thin-shell mirror substrates for x-ray astronomy, David L. Windt...following talk 


\section{Process Sequence}

Surface profile metrology

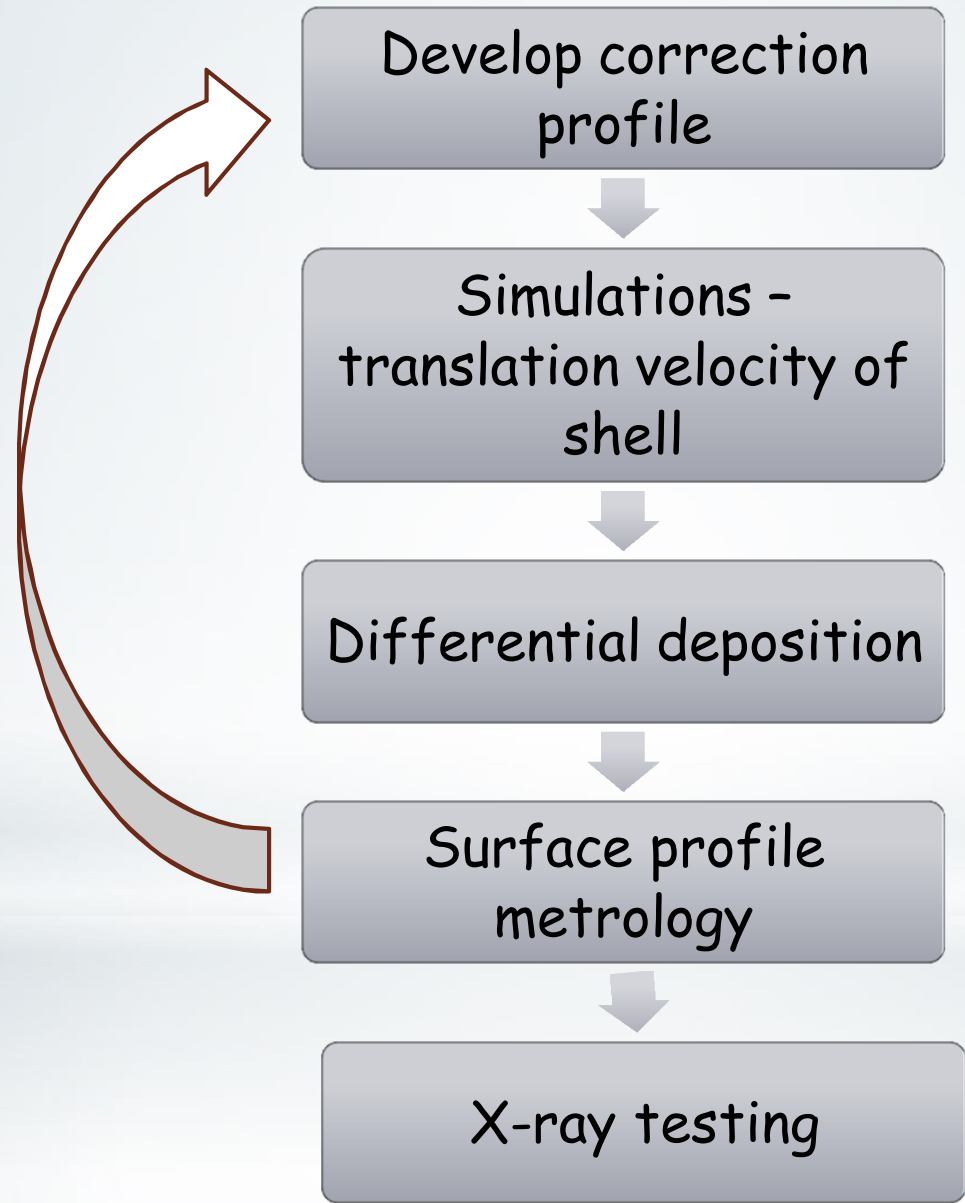




\section{Proof of concept on miniature optics}

An existing vacuum

chamber was modified for the proof of concept on the miniature optics developed for radio-nuclide imaging
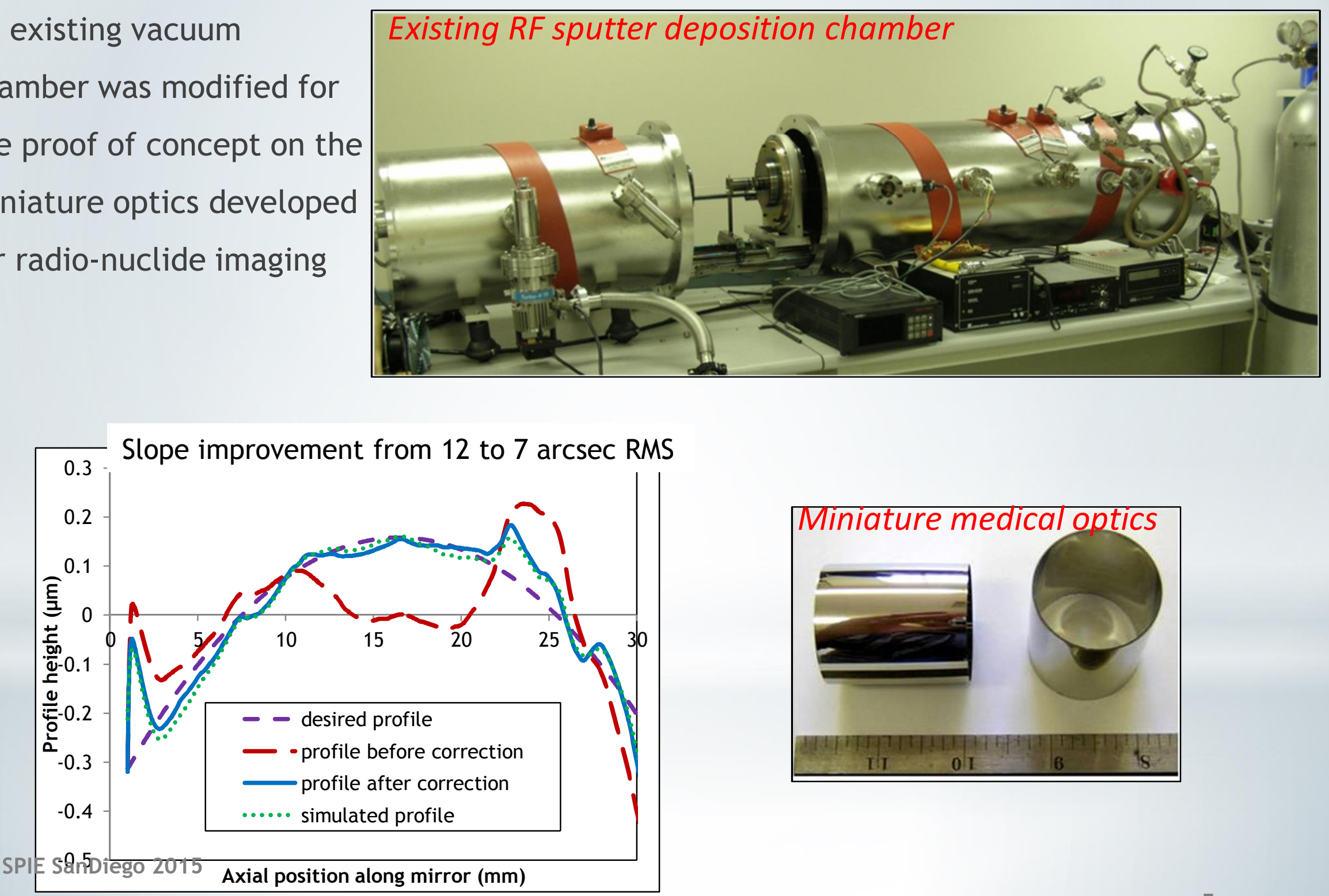


\section{Coating Systems (DC magnetron)}

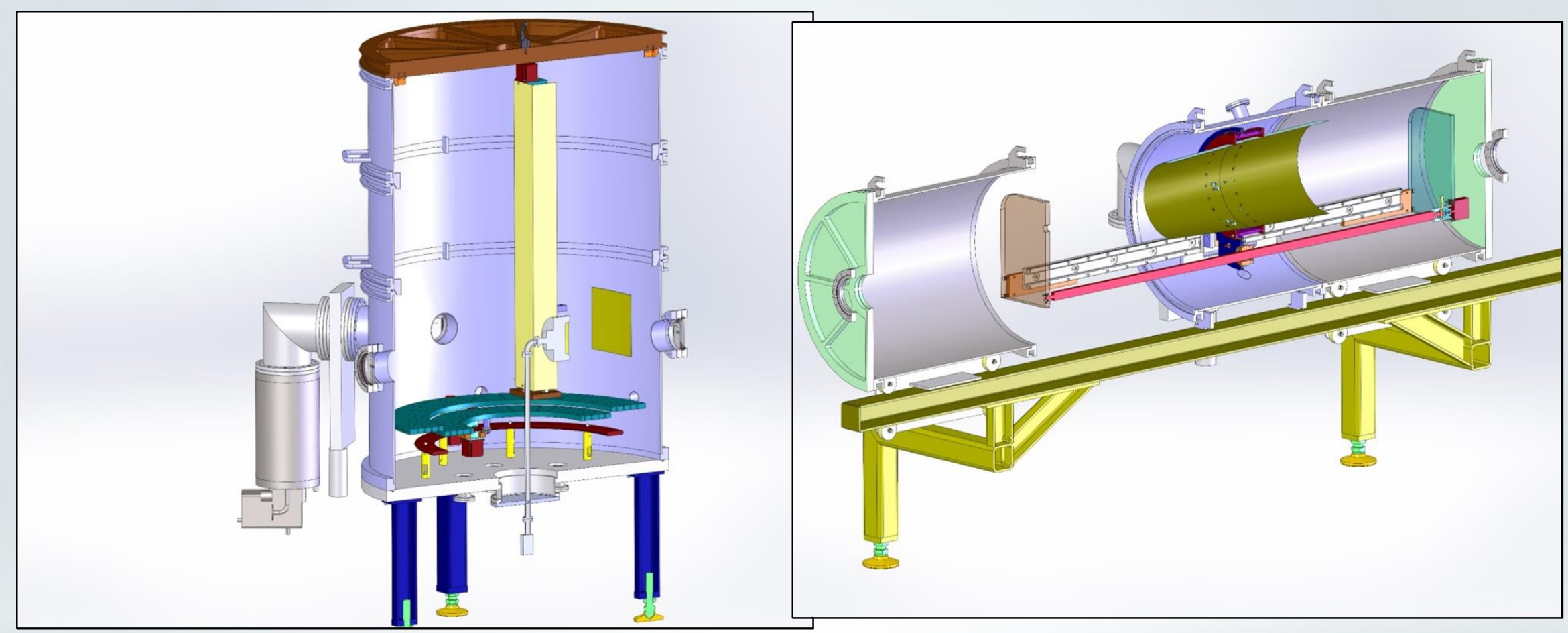

Vertical chamber for segmented optics and very large full shell optics (>0.5m diameter)
Horizontal chamber for $0.25 \mathrm{~m}$ diameter and up to $0.6 \mathrm{~m}$ length - scale full shell optics 


\section{Coating Systems}
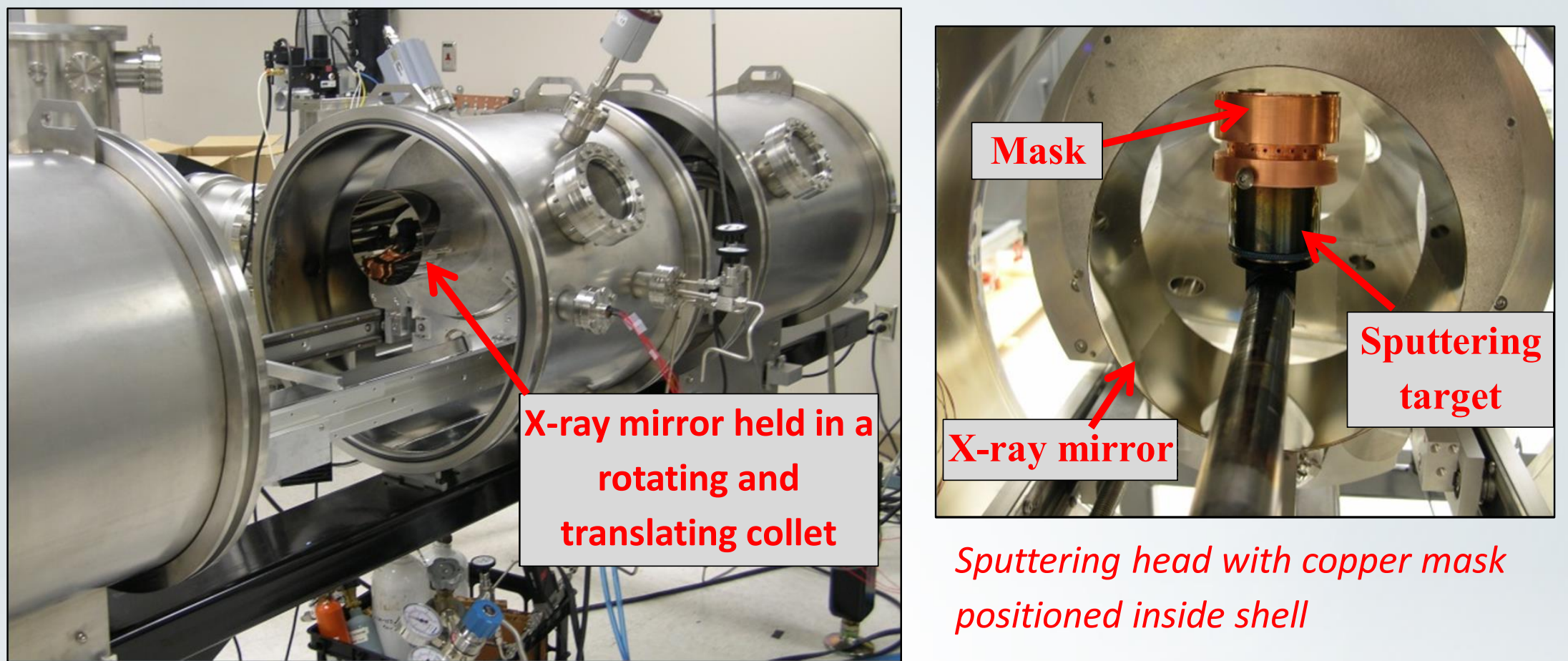

Sputtering head with copper mask positioned inside shell

Horizontal differential-deposition chamber

* For full-shell cylindrical optics

* Oriented horizontally - mounted on rail system - splits into 3 section for easy access

* Computer controlled translation and rotation stages with encoders

* Matlab - GUI interface to control the stages

SPIE SanDiego 2015 


\section{Coating Systems}

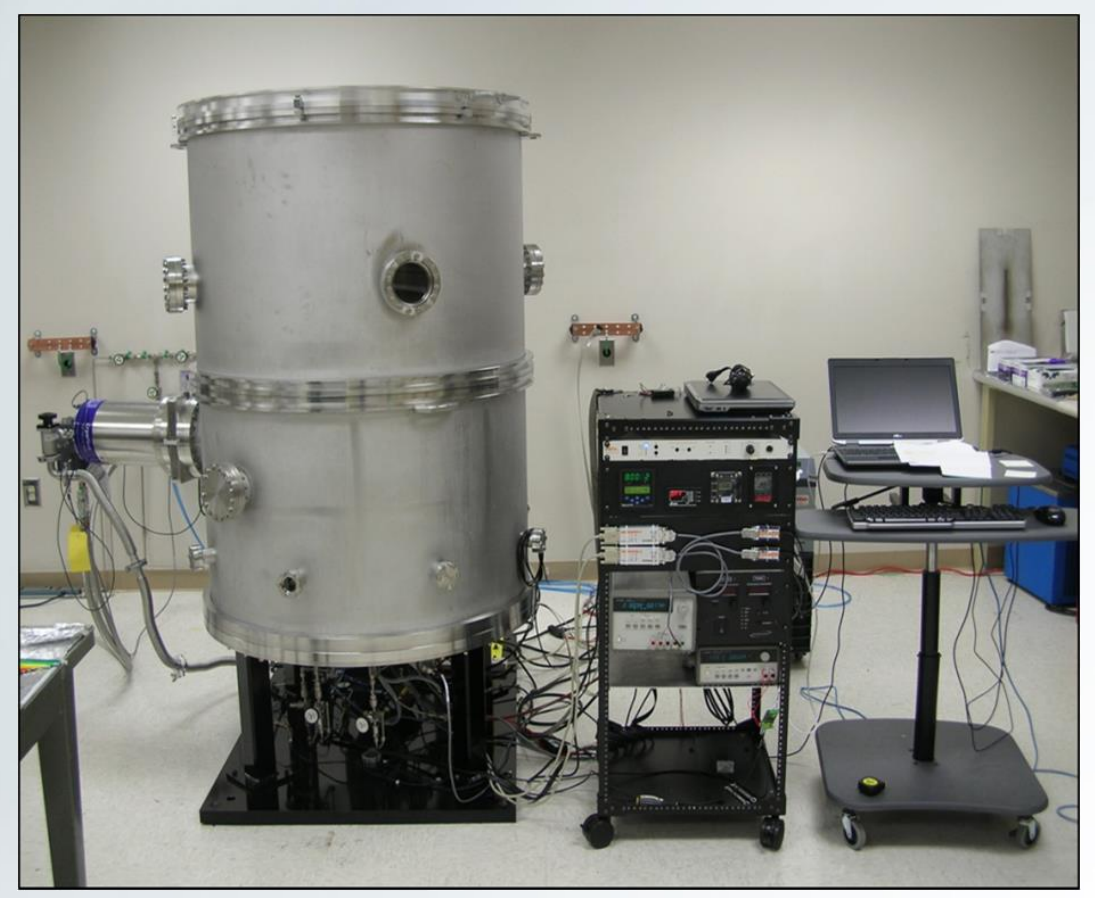

Vertical deposition chamber

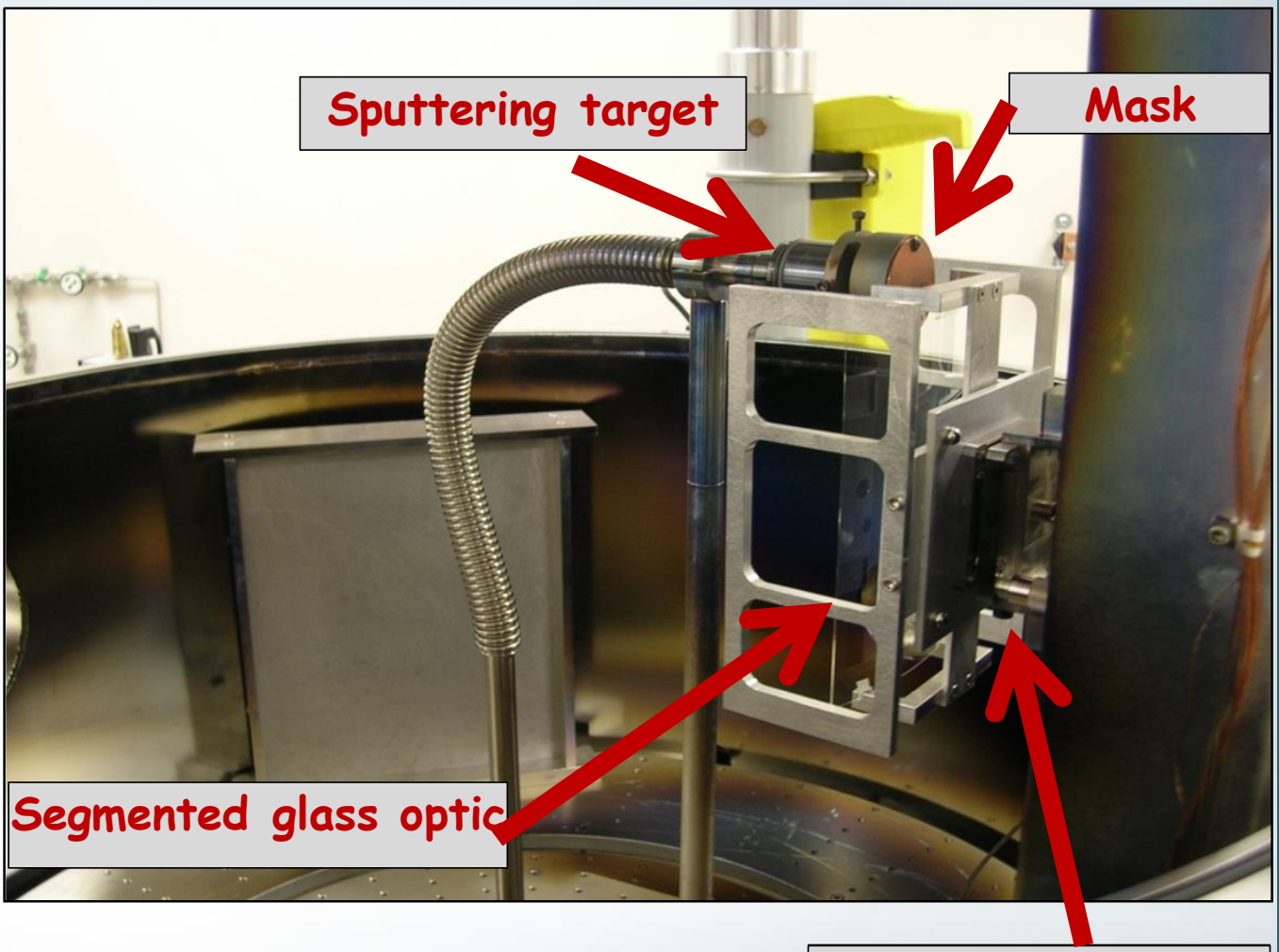

Translation stage

* Details in the upcoming talk by Dr. Carolyn Atkins

* Optimization of coating parameters

* Good estimate of sputter beam profile for various slit-widths 


\section{Test Coatings}

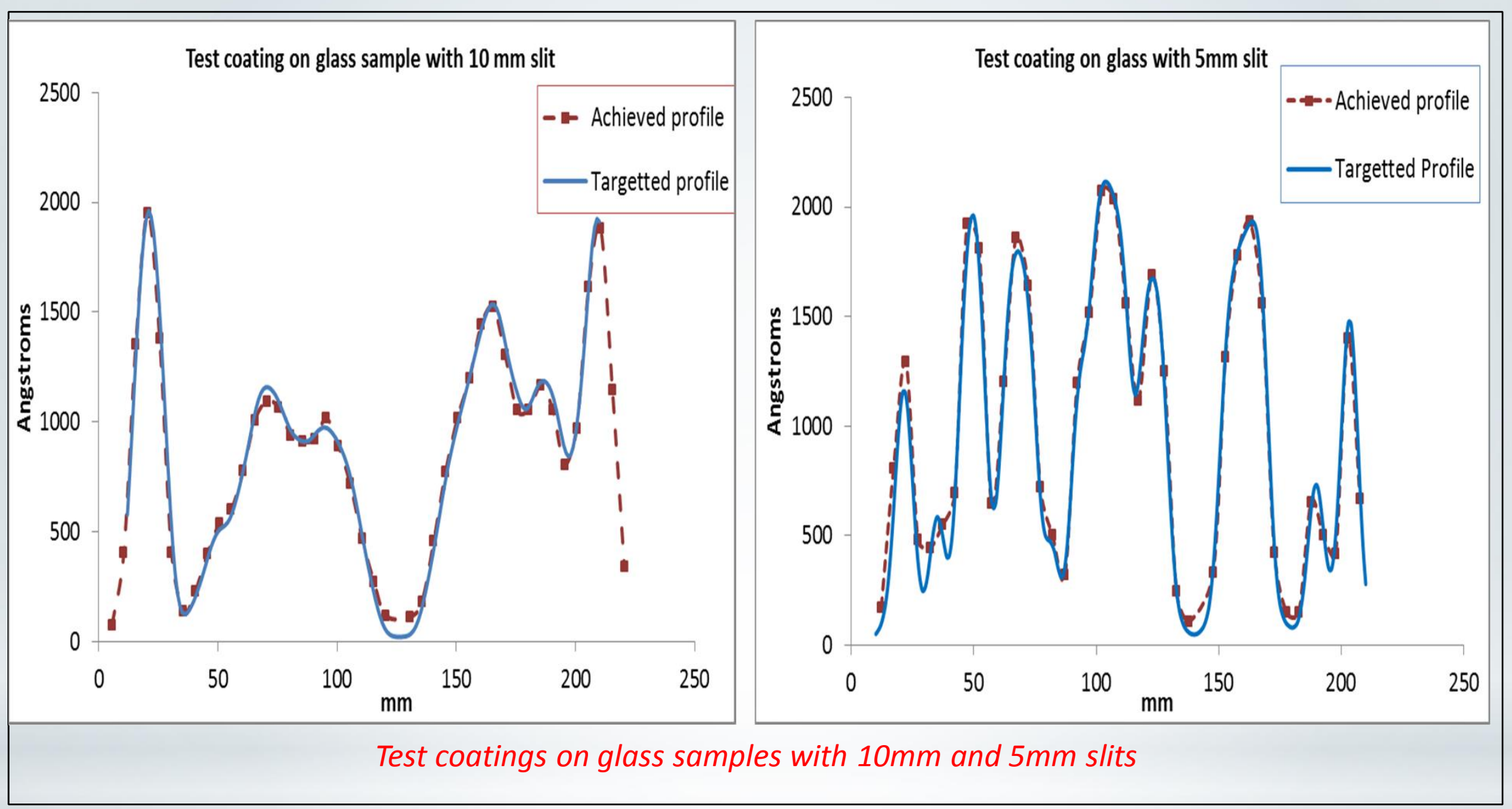

* KLA-Tencor step profiler is used to measure the coating thickness on glass samples

* Good agreement with simulations 


\section{Metrology - VLTP}

* Vertical Long Trace Profiler

* $1 \mathrm{~mm}$ spatial interval

* New 2D camera and modified software

*Established procedures to obtain repeatability of $<100$

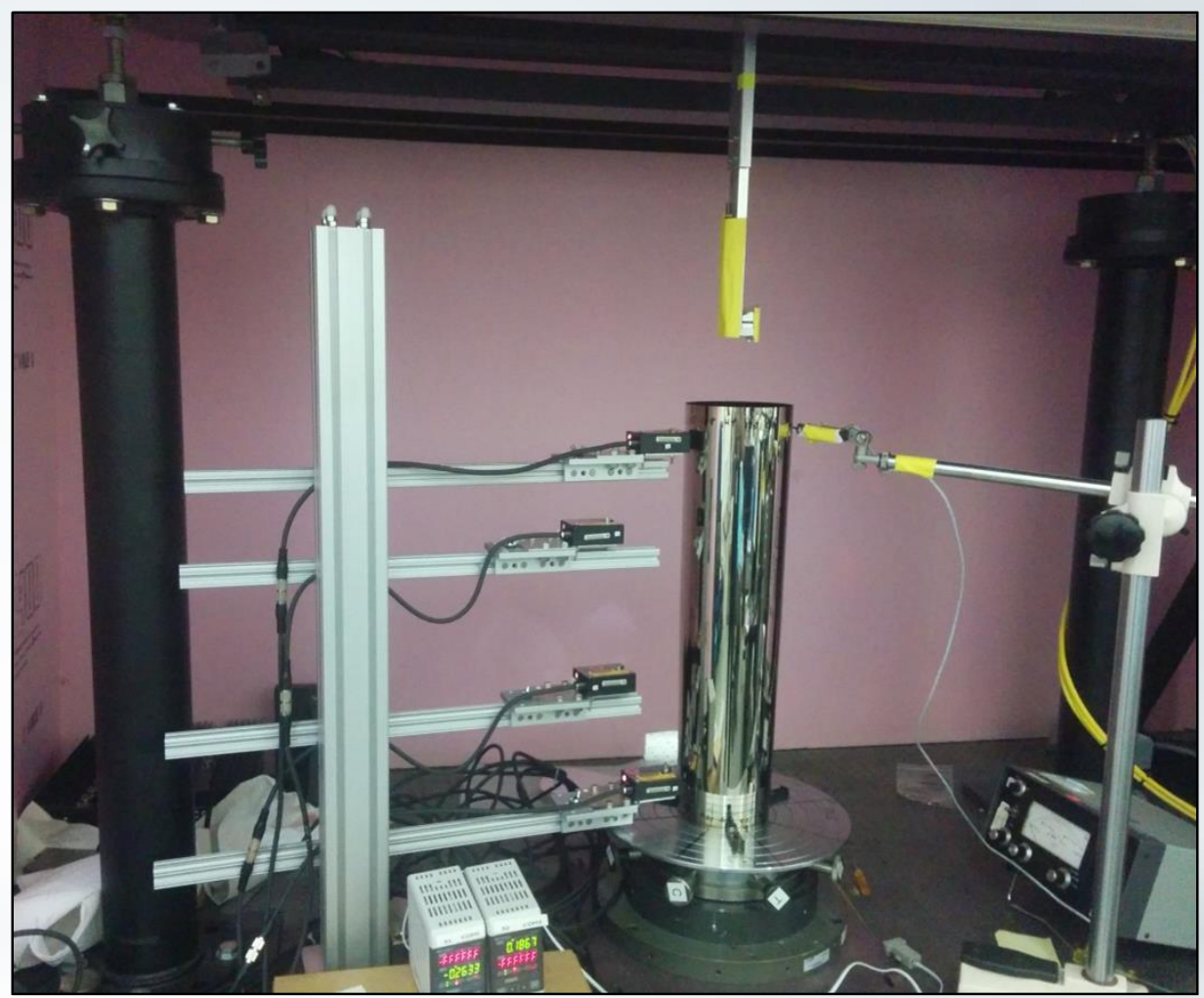
Angstroms 
$150 \mathrm{~mm}$ diameter shell - single meridian; pre- and post- two stages of correction

\section{- high frequencies only}
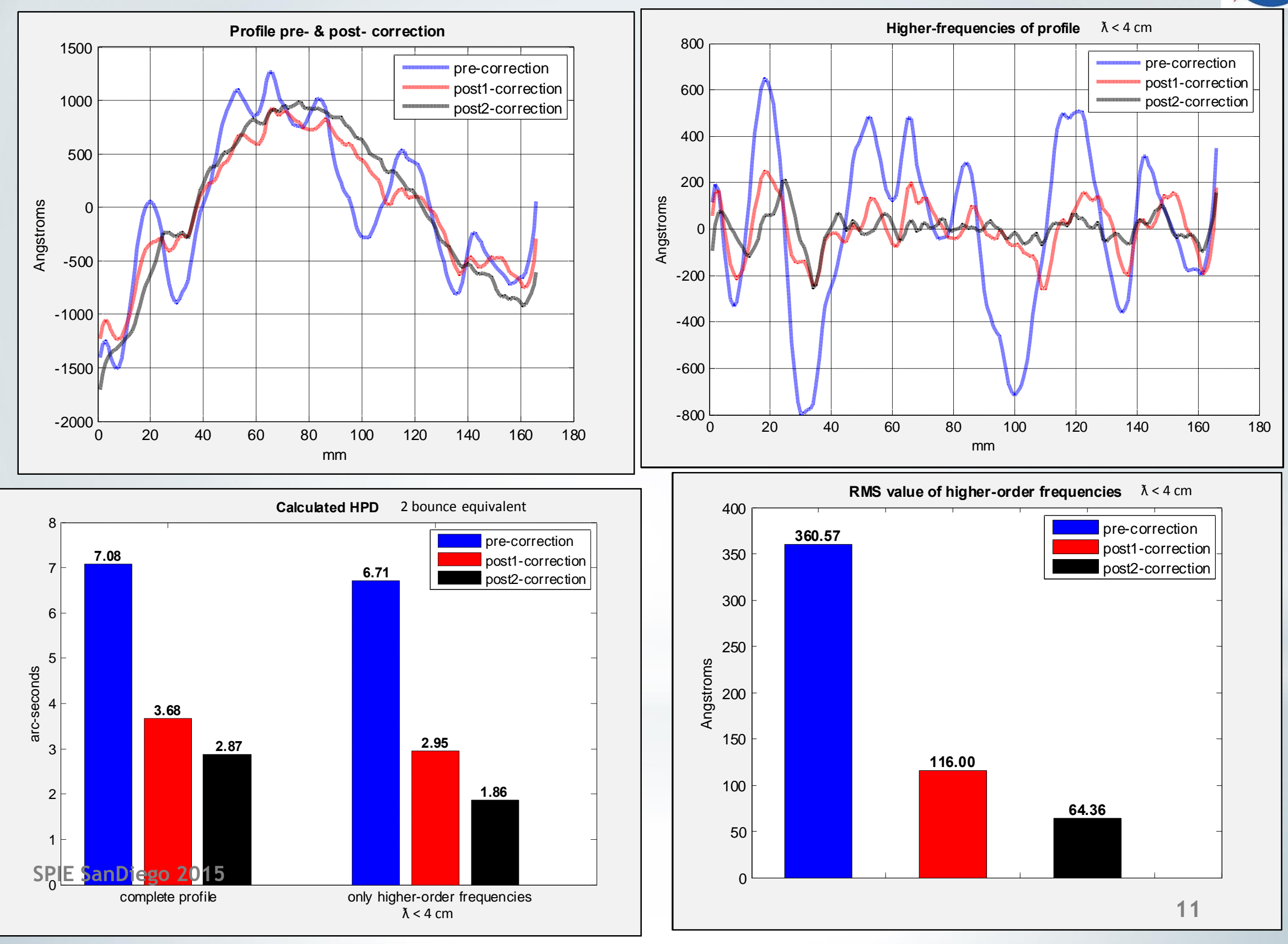
Higher-frequencies complete full-shell - average of all meridians

* Higher-frequencies of individual meridians are similar in deviations - replicate from the mandrel

* Average of all meridians $-1^{\text {st }}$ stage of correction

* $2^{\text {nd }}$ stage of correction is better achieved with specialized correction at first stage
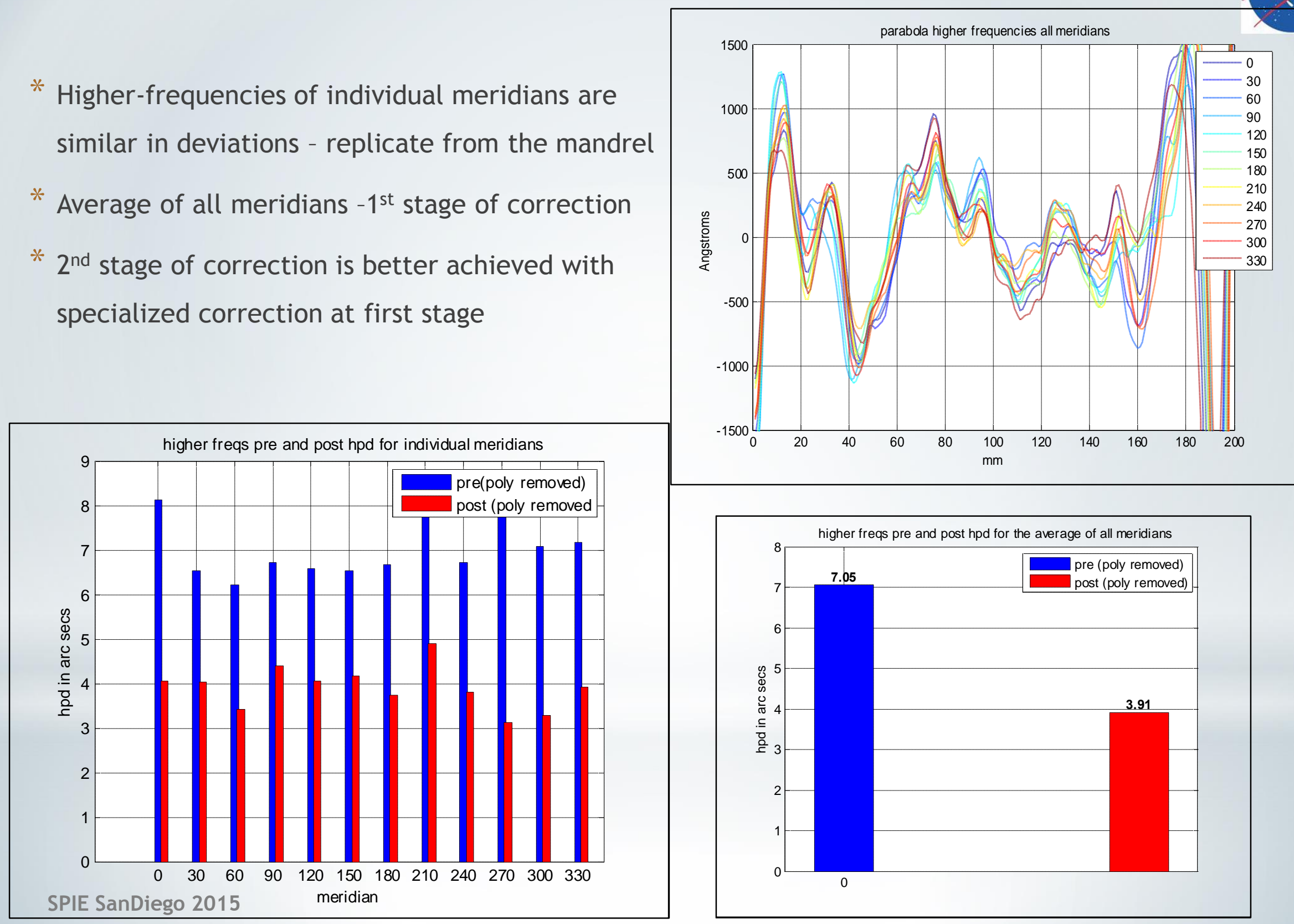


\section{$\underline{\mathrm{X} \text {-ray testing - pre-and post- differential coating }}$}
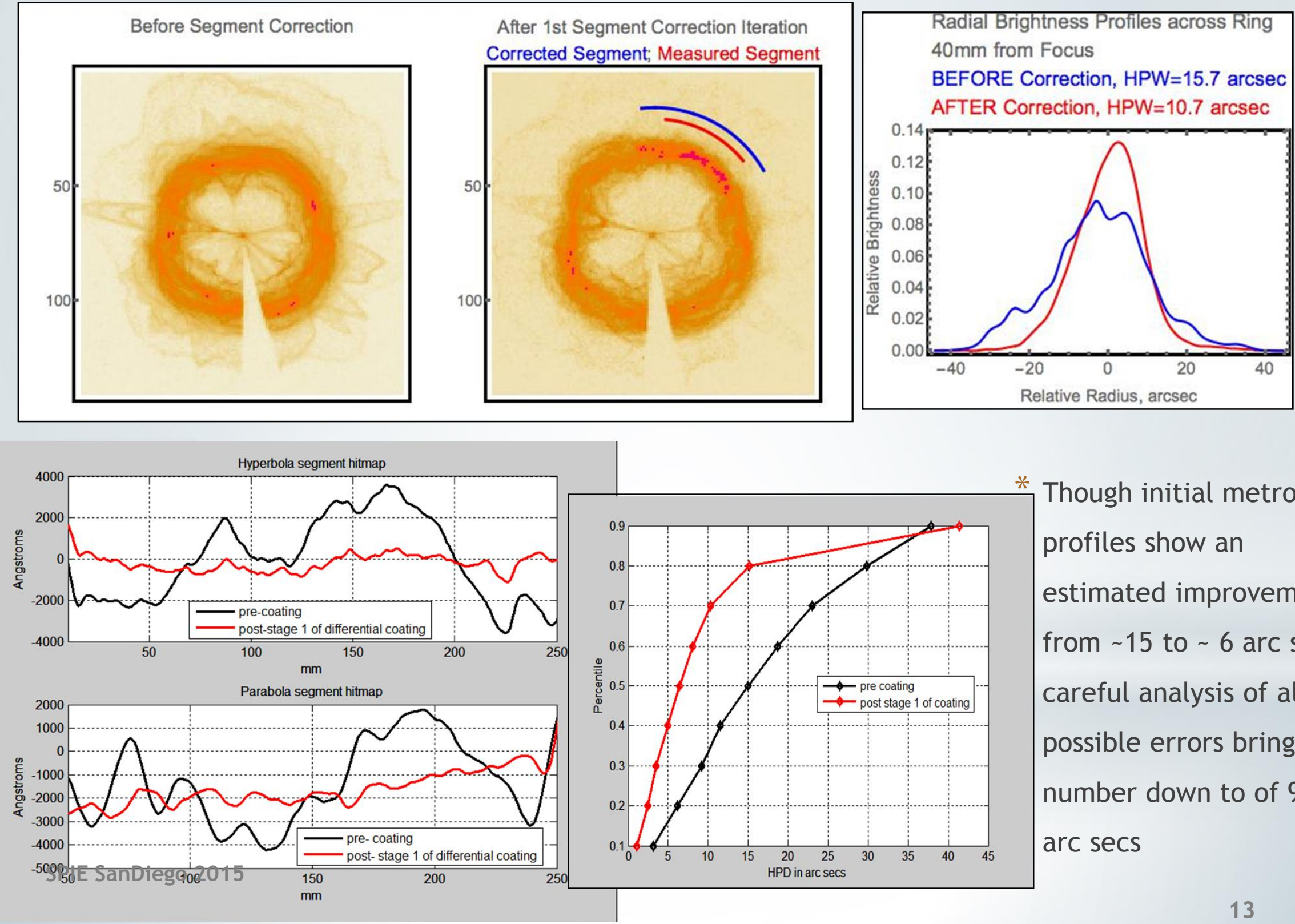

Though initial metrology profiles show an estimated improvement from $\sim 15$ to $\sim 6$ arc secs, careful analysis of all the possible errors bring this number down to of 9.23 $\operatorname{arc}$ secs 

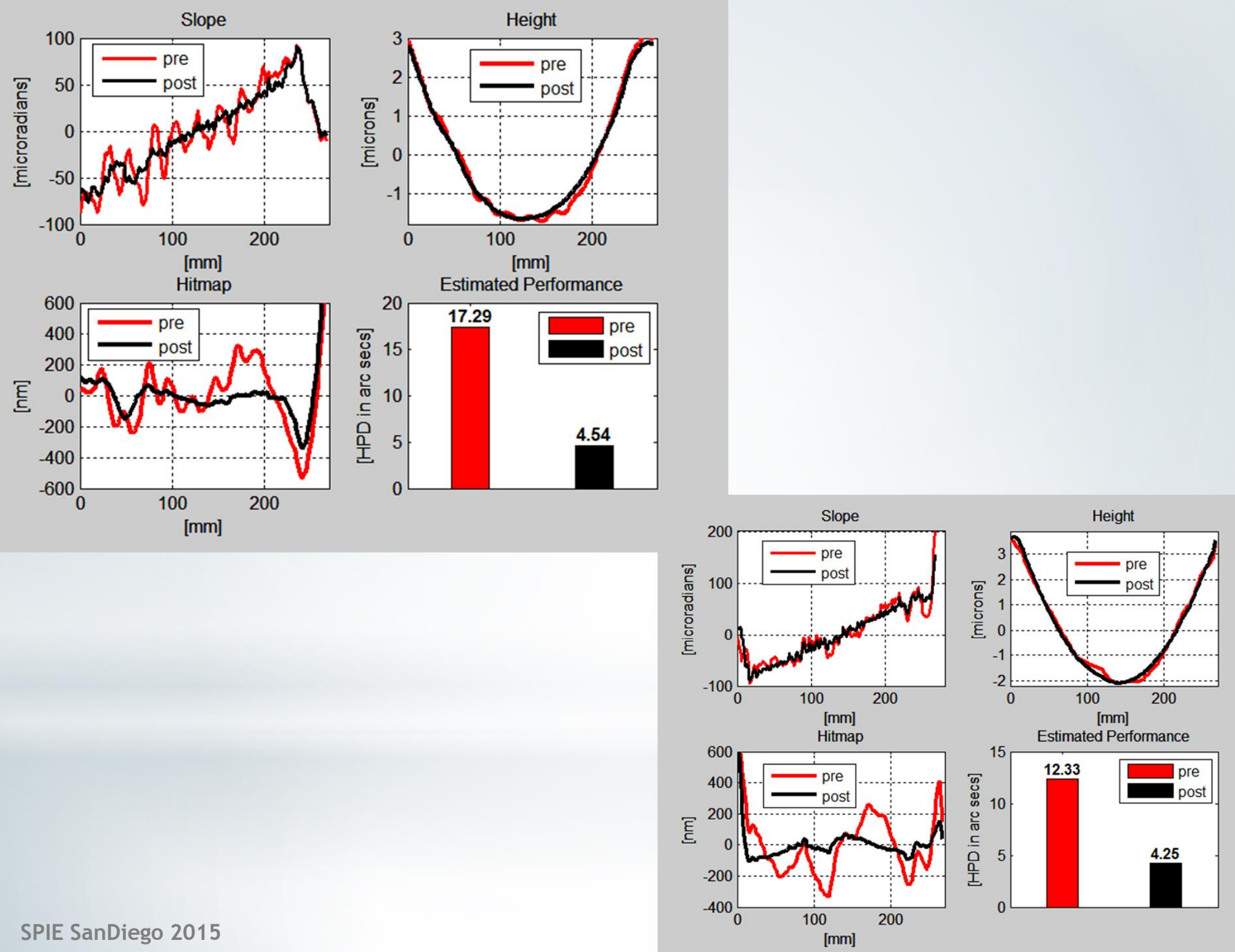


\section{Possible Sources of Errors - Improvements}

* Variation of sputtered beam profile along the length of mirror - particularly for short focal length mirrors - Improvements in mechanical set-up

* Thorough characterization of the overlap areas in the case of customized correction for each meridian

* Improvements in the mask to shell alignment system

* Stress effects - Quantify and control stress 


\section{Coating Stress Measurement System}
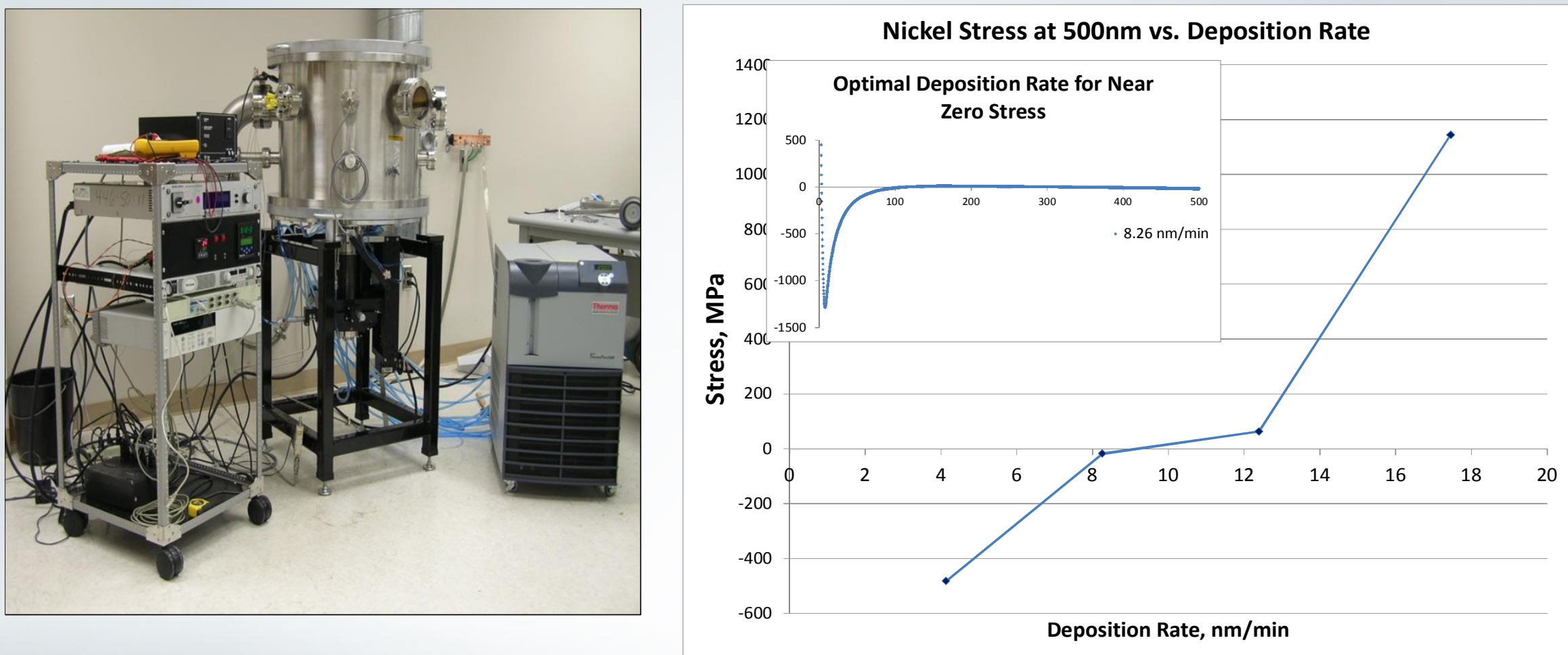

* Simulations show that for full shell optic need < 10MPa stress to get <

1 arcsec optic (dominated by longer-wavelength corrections). Set up dedicated system to characterize coating stresses. 


\section{Conclusions}

*Advantages -

* Can be used on any type of optic, full-shell or segmented, mounted or unmounted

* Can be used to correct a wide range of spatial errors

* Could be used in conjunction with other techniques... e.g. active optics

*Efforts are in progress to achieve the best possible improvement with differential deposition and to quantify the improvement with X-ray testing 